\title{
The Potential of Hybridising Interactive Eye Tracking Technology with Decision Support in Medical Image Interpretation
}

\author{
Laura McLaughlin \\ Ulster University \\ Northern Ireland \\ mclaughlin-116@ulster.ac.uk
}

\author{
Raymond Bond \\ Ulster University \\ Northern Ireland \\ rb.bond@ulster.ac.uk
}

\author{
Jonathan McConnell \\ NHS Greater Glasgow and Clyde \\ Scotland \\ jonathan.mcconnell@ggc.scot.nhs.uk
}

\author{
Ciara Hughes \\ Ulster University \\ Northern Ireland \\ c.hughes@ulster.ac.uk
}

\author{
Sonyia McFadden \\ Ulster University \\ Northern Ireland \\ s.mcfadden@ulster.ac.uk
}

\begin{abstract}
This paper explores the use and future of interactive eye tracking technology within medical image interpretation. Eye tracking technology gives us an insight into the process and approaches taken by a reporting clinician during image interpretation. Application of this technology with verbalisation of thought processes and artificial intelligence could progress education and image interpretation further.
\end{abstract}

Eye tracking, education,

\section{INTRODUCTION}

Eye tracking has frequently been used in research across a mass of disciplines (Bond et al. 2014; Currie et al. 2017; McLaughlin et al. 2017). It has been used to help understand the process of image interpretation and to assess and provide feedback/training on the interpretation process. Feedback based on eye tracking technology has led to improvements in performance.

There is no standard systematic approach to chest image interpretation. Previous research has provided training and assessed the impact (Donovan et al. 2008; Litchfield et al. 2010). Improvements in the performance of participants post implementation of this feedback were recognised. Perceptually based feedback led to a $16 \%$ increase in observer performance compared to showing the observer the image again with no eye tracking feedback highlighted (Krupinski et al. 2000). Often trainee radiologists and reporting radiographers combine advice given in interpretation guidance and a variety of recommended search techniques to form their own search strategy in image interpretation.

A systematic method of interpreting the entire image would limit the possibility of misdiagnosis or failure to detect a pathology (Del Ciello et al. 2017). learning, medical image interpretation

A training tool for laparoscopic surgery which consisted of the eye gazes of experienced surgeons projected onto a trainees screen improved completion times and reduced errors; however such training tools have yet to be formed for use in radiology (Chetwood et al. 2012). By studying the thought process of an expert radiologist's image interpretation, we formed a digital tool with the aim of providing a uniform standard of training which optimises learning by using the consultant radiologists' and reporting radiographer's experience and technique.

\section{INTERACTIVE TRAINING TOOL}

\subsection{Search strategy training tool}

The search strategy comprises of a series of questions and prompts to guide the user to exclude pathologies, systematically search the image and form a diagnosis (McLaughlin et al. 2017). The search strategy begins by allowing reporting clinicians to focus on the 'general considerations' of the image presentation. Following this initial image analysis prompt, the search strategy leads the user through the image and encourages them to 
The Potential of Hybridising Interactive Eye Tracking Technology with Decision Support in Medical Image Interpretation McLaughlin • Bond $\bullet$ McConnell $\bullet$ Hughes $\bullet$ McFadden

consider different parts of the image individually. The search strategy comprises six sections;

- General image considerations

- Tubes/lines/devices
- Bony thorax/soft tissues

- Diaphragm/heart/mediastinum

- Lung zones

- Lung shadows

\section{The diaphragm, heart and mediastinum}

\section{Assess the diaphragm, heart and mediastinum}

a. Any free air?

b Is the cardiothoracic ratio?

c. Is the gortic knuckle

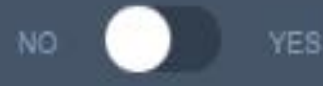

$350 \% \quad 300 \%$

NOGMAL ( ) AENGEMAI
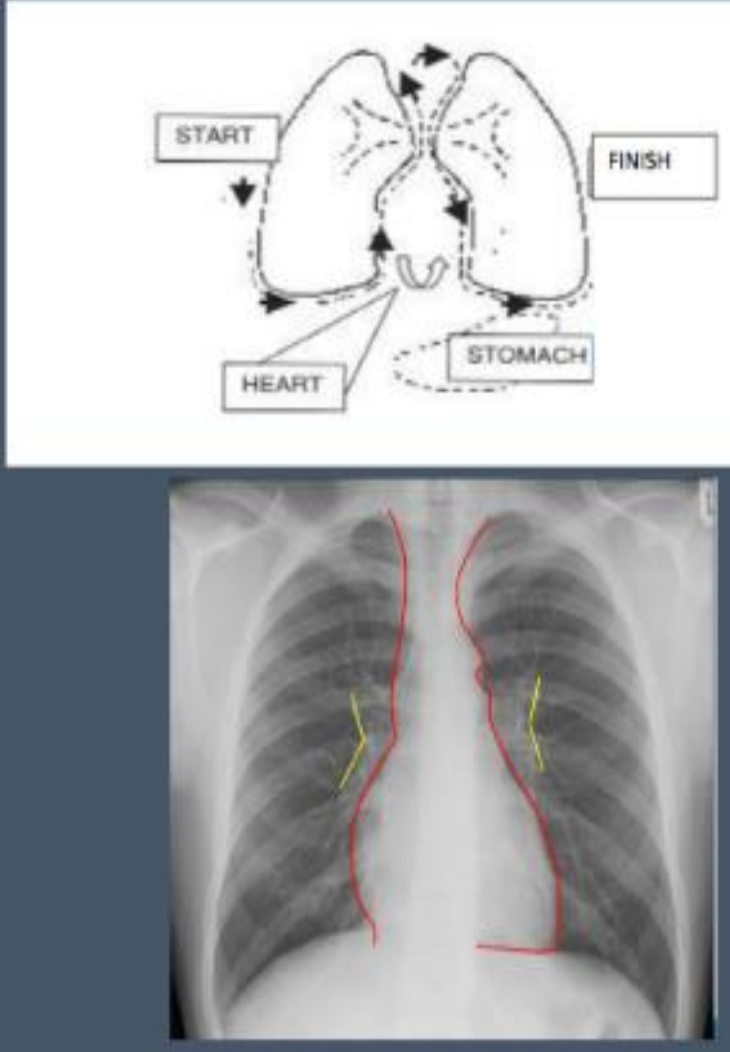

Figure 1: An example of the questions and diagrams included within the online training tool. 


\subsection{Educational tool}

The educational tool consists of videos comprised of expert eye gazes and scan paths recorded during chest image interpretation and collected whilst the expert used the search strategy training tool. Expert input was from qualified reporting clinicians who specialise in chest image interpretation.

The expert's eye gaze behaviours were recorded as well as the verbalisation of their thought processes during their interpretation which provides a clear description of their search strategy. The training tool, once finalised, was transformed into an online digital format for participant's ease of use.

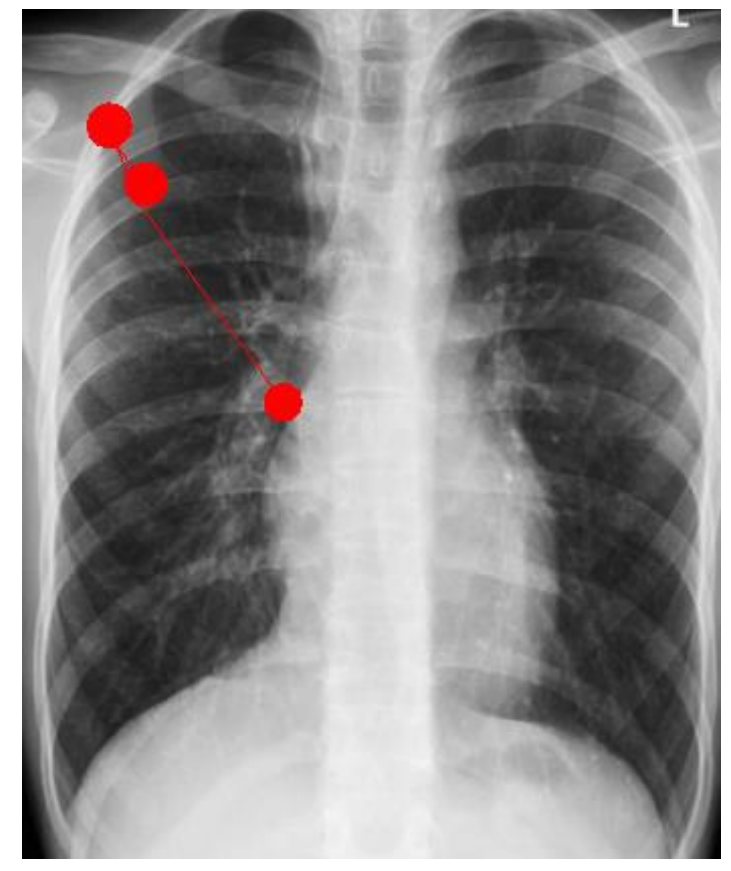

Figure 2: An example of the eye tracking technology used within the digital training tool

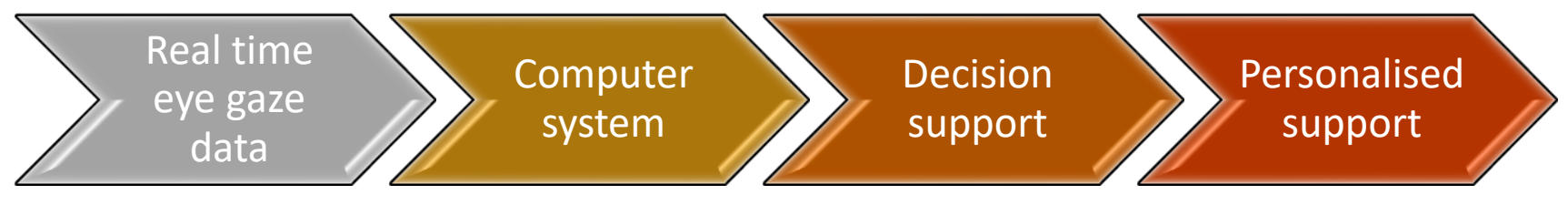

Figure 3: Real time eye gaze data could inform a computer system of interpreter uncertainty, confidence, competence and areas that were not appropriately interrogated - this coupled with decision support could be powerful personalised support.

\section{FURTHER USE}

Eye tracking technology could play an important role in developing and improving image interpretation techniques. By encouraging reporting clinicians to systematically search the entire image, the technology could be central to reduce errors and delays in patient diagnosis and care. The digital training tool described here provided users with examples in the form of experts eye gazes during a systematic search of chest radiographic images. Personalised and rapid eye tracking feedback directly following/during a participant's image interpretation process could substantially improve the interpretation techniques. Highlighting areas missed visually, by a participant could lead to improvements in interpretation.
By analysing eye tracking data and developing an algorithm to assess areas in the image fixated on most commonly and those areas missed, software could be programmed to highlight areas of the image which were not fixated on by a reporting clinician and which the computer deems to be "an irregular area" within the image. This involves the development of an automated data visualisation tool to show unstudied areas on the image by comparing the image with a template of image shape and contrast/density changes that represent a pathology in the lung fields. Separate algorithms could be developed using machine learning approaches that use real-time eye gaze to infer the clinician's level of certainty and competency (Figure 3 ). Providing an overview of the areas which were not fixated on by the reporting clinician when interpreting an image which the computer believes to be suspicious for the presence of a pathology, 
could also provide decision support and suggestions.

There are various directions that this technology could take in the world of medical image interpretation research. Real time eye tracking feedback combined with artificial intelligence, whereby the reporting clinician would be learning and receiving feedback immediately during the image interpretation process, could greatly change this clinical role. As technological advances are made, the use of digital systems could lead the way in identifying areas which are not fixated on during an image interpretation to the image viewer. Advances such as these would maximise rigour and ensure image interpretations are thorough, possibly contributing to the reduction of errors and enhancement of image interpretation accuracy.

\section{REFERENCES}

Bond, R. Zhu, T. Finlay, D. Drew, B. Kligfield, P. Guldenring, D. Breen, C. Gallagher, A. Daly, M. and Clifford, G. (2014) Assessing computerized eye tracking technology for gaining insight into expert interpretation of the 12-lead electrocardiogram: an objective quantitative approach. Journal of Electrocardiology, 47(6), 895906.

Chetwood, A.S., Kwok, K.W., Sun, L.W., Mylonas, G.P., Clark, J., Darzi, A. and Yang, G.Z., 2012.

Collaborative eye tracking: a potential training tool in laparoscopic surgery. Surgical endoscopy, 26(7), 2003-2009

Currie, J. Bond, R. McCullagh, P. J. Black, P. Finlay, D. and Peace, A. (2017) Eye Tracking the Visual Attention of Nurses Interpreting Simulated Vital Signs Scenarios: Mining Metrics to Discriminate Between Performance Level. IEEE Transactions on Human-

Machine Systems,. pp. 1-11. [Journal article]

Del Ciello, A. Franchi, P. Contegiacomo, A. Cicchetti, G. Bonomo, L. Larici, A. R. (2017). Missed lung cancer: when, where, and why? Diagnostic and Interventional Radiology, 23(2), 118-126. http://doi.org/10.5152/dir.2016.16187

Donovan, T. Manning, D.J. and Crawford, T. (2008) Performance changes in lung nodule detection following perceptual feedback of eye movements. Medical Imaging, 691703-9.
Krupinski, E.A. (2000) The importance of perception research in medical imaging. Radiation Medicine, 18(6), 329-334.

Kundel, H.L. Nodine, C.F. and Krupinski, E.A. (1990) Computer-displayed eye position as a visual aid to pulmonary nodule interpretation. Investigative Radiology, 25(8), 890896.

Litchfield D. Ball L.J. Donovan T. Manning D.J. and Crawford, T. (2010) Viewing another person's eye movements improves identification of pulmonary nodules in chest $\mathrm{x}$-ray inspection. Journal of Experimental Psychology: Applied, 16(3), 251-262.

McLaughlin, L. Bond, R. Hughes, C. McConnell, J. McFadden, S. (2017) Computing Eye Gaze Metrics for the Automatic Assessment of Radiographer Performance during X-ray Image Interpretation, International Journal of Medical Informatics. 11-21

McLaughlin, L. Woznitza, N. Cairns, A. McFadden, S. Bond, R. Hughes, C. Elsayed, A. Finlay, D. McConnell, J. (2018) Digital training tool for interpreting radiographic images of the chest, Radiography 